\title{
The multi-objective optimization of tunneling boring machine control based on geological conditions identification
}

Hongyuan Wang and Jingcheng Wang

\author{
Department of Automation, Shanghai Jiao Tong University, Shanghai, China
}

\begin{abstract}
Purpose - The purpose of this paper aims to design an optimization control for tunnel boring machine (TBM) based on geological identification. For unknown geological condition, the authors need to identify them before further optimization. For fully considering multiple crucial performance of TBM, the authors establish an optimization problem for TBM so that it can be adapted to varying geology. That is, TBM can operate optimally under corresponding geology, which is called geology-adaptability.

Design/methodology/approach - This paper adopted $k$-nearest neighbor (KNN) algorithm with modification to identify geological conditions. The modification includes adjustment of weights in voting procedure and similarity distance measurement, which at suitable for engineering and enhance accuracy of prediction. The authors also design several key performances of TBM during operation, and built a multiobjective function. Further, the multi-objective function has been transformed into a single objective function by weighted-combination. The reformulated optimization was solved by genetic algorithm in the end.

Findings - This paper provides a support for decision-making in TBM control. Through proposed optimization control, the advance speed of TBM has been enhanced dramatically in each geological condition, compared with the results before optimizing. Meanwhile, other performances are acceptable and the method is verified by in situ data.

Originality/value - This paper fulfills an optimization control of TBM considering several key performances during excavating. The optimization is conducted under different geological conditions so that TBM has geological-adaptability.
\end{abstract}

Keywords Tunnel boring machine, KNN, Geological identification, Multi-objective optimization, Weight adjustment, Geological adaptability

Paper type Research paper

\section{Introduction}

Tunnel boring machine (TBM) is a complex special equipment used for tunnel excavating, which integrates cutting of surrounding rock, slagging, hole formation, grouting and auxiliary system for consecutive excavation operations. It is involved with mechanical engineering, control engineering, materials engineering and electric engineering as an aggregation. Compared with traditional digging methods, this equipment has obvious advantages on such as safety and reliability, fast digging speed, low impact on the

(C) Hongyuan Wang and Jingcheng Wang. Published in Journal of Intelligent Manufacturing and Special Equipment. Published by Emerald Publishing Limited. This article is published under the Creative Commons Attribution (CC BY 4.0) licence. Anyone may reproduce, distribute, translate and create derivative works of this article (for both commercial and non-commercial purposes), subject to full attribution to the original publication and authors. The full terms of this licence may be seen at http:// creativecommons.org/licences/by/4.0/legalcode

This work is supported by National Natural Science Foundation of China (No. 61533013, 61633019), Key projects from Ministry of Science and Technology (No. 2017ZX07207003, 2017ZX07207005), Shaanxi Provincial Key Project (2018ZDXM-GY-168) and Shanghai Project (17DZ1202704). 

$\underset{1,1}{\text { JIMSE }}$ surrounding environment of construction and low comprehensive cost. It has been widely used in many infrastructure constructions since it appears, including subway tunnels, water conservancy, railway tunnel and even in national defense facilities for each country. Therefore, the state-of-the-art technologies integrated in this special equipment have received many attentions from academic community and industrial enterprises. In industry, famous manufacturers include China Railway Engineering Equipment Group, Robbins, Herrenknecht, Mitsubishi Heavy Industries, Komatsu. However, there still exists many challenges in TBM tunneling operation that need to be settle at present, see Zheng et al. (2016) and Home (2016).

Reasonable setting of TBM control parameters for tunneling (mainly focus on advancing speed and rotation speed of cutter-head) is a critical section concerning the success of the whole project, especially for the compound or adverse geology (Gong et al., 2016). From an engineering point of view, at present, the way to adjust control parameters of TBM is almost executed by the driver according to their experience. Such a manner brings a too conservative decision-making that slows down the efficiency of excavating. Current research studies on TBM control and the way to automatically adjustment of control parameters under guaranteeing performance, are very limited and immature. Liu et al. (2011) have proposed the multidrive synchronization control based on the ring coupling strategy for driving systems of cutter-head. Rostami (2016) has investigated some performances of TBM that may need to be considered and their predictions in order to understand the geological conditions more precisely. However, this paper does not discuss about how these performances can be utilized for TBM control. Yang et al. (2019) designed a distributed model predictive control scheme to implement the tracking control of TBM, where the performance only considered about the error between actual trajectory and reference as like the common setting in model predictive control. The works in Zhao et al. (2015) introduced a fuzzy Proportion Integration Differentiation (PID) controller for each induction motor. Combined with master-slave control strategy, the robustness has been enhanced for compound geological condition. Gao et al. (2019) utilized numerous in situ data to construct deep neural network with different construct for predicting tunneling parameters. The accuracy of prediction is desirable and notable, but the actual value is not necessarily optimal for TBM. Besides, this works did not consider related constraints, and the predictive values may violate the constraint, which are not be allowed in practical. On the other hand, all abovementioned works assume that the geological condition has been already known before design. On the other hand, it can be seen that above works do not consider the performance of TBM during the operation. That is, the control signals obtained from those methods are not the optimal one for TBM during tunneling. Even there are some related works involved with optimization, those mainly focus on the optimization for parameter predictions rather than control. These issues motivate us to pay attention to the optimization of TBM control where may exist several performances at the same time and even without accurate information about geological condition.

The main contributions of this paper lies in: (1) Before control design of TBM, we identify geological condition using $k$-nearest neighbor (KNN) method based on in situ data. The results will be utilized in the later optimization problem formulation; (2) Several crucial performances of TBM during the excavating are presented, which will be used as objective function in later formulation of optimization problem; (3) A multi-objective function is formulated for TBM control. Furthermore, we convert established multi-objective optimization problem into single-objective optimization problem with setting well-matched weighted factors corresponding identified different geological conditions. The proposed methods can be able to make TBM be adapted to different geological condition; (4) A case study is investigated to verify the effectiveness of proposed method based on the in situ data and parameters. 
This paper is organized as follow. The necessary research backgrounds are stated in Section 2. In Section 3, geology identification is implemented by using KNN method based on the in situ data. In Section 4, several effective performance functions of TBM in excavating are presented and a multi-objective optimization problem is formulated with specified constraints. Moreover, multi-objective optimization problem is transformed to singleobjective optimization problem and solved by using genetic algorithm. A case study is illustrated to verify the efficacy of proposed method in Section 5 and conclusions are drawn in Section 6.

\section{Research background}

\subsection{Tunnel boring machine}

Before illustrating our studies, this section will introduce some necessary research backgrounds in this paper. First, we briefly show the basic structure of TBM in this section, followed by the motivations of this paper. Figure 1 gives a sketch about main part of TBM excluding auxiliary support system. A number of disc cutters are installed on the cutterhead. There are several buckets around the edge of cutter-head used to collect the rock slag, so that the rubbles can be transferred by built-in conveyor belt. Shield is used to prevent cutterhead from stuck caused by rubbles during the excavating process. The main driving system is composed of multiple induction motors through the gear fitting to the planetary gear. The induction motors provide sufficient force during the excavating for the cutter-head. Main beam (or main girder), as a bridge, is connected with cutter-head, shield, bracing boots, propulsion system and auxiliary support system. It can transfer the advance force produced by the hydraulic cylinder and the torque produced by the multiple motors in the cutter-head. This will achieve the advancement and step-changing operation. As a part of the propulsion system, the hydraulic cylinders located on the two sides of main girder will push TBM forward along the specified trajectory. Auxiliary support system, including water supply system, power supply system, electrical equipment, transport units and so on, will provide necessary assistance for consecutive excavating.

The main driving system (or called cutter-head systems in some literatures) of TBM is mainly composed of cutter-head, hobs, motors, pinions, large gears and reduction gear box. It is controlled by a number of driving motors, where the number of motor depends on the radius of cutter-head. Plate 1 illustrates the real product and the main structure of driving system. The dynamics model of driving systems of TBM can be found in Li et al. (2013), which is omitted here due to the limitation of space. Due to the fact that the driving system directly touch the excavating surface, its control will influence the equipment performance, or even the efficiency of the whole project.

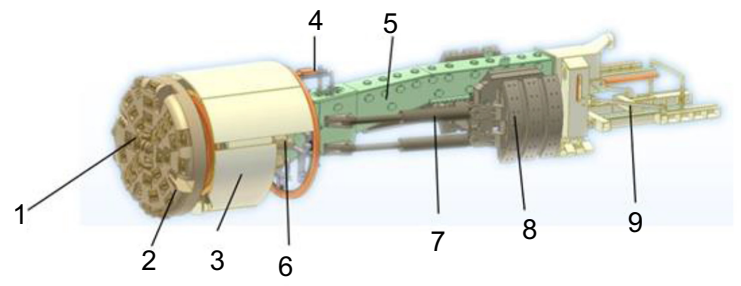

1-Disc cutter; 2-Cutter-head; 3-Shield; 4-Tapping; 5-Main beam; 6-Induction motor; 7-Hydraulic cylinder; 8-Horizontal bracing boots; 9-Back-up equipment
Optimization

of TBM with geology recognition

89 


\section{JIMSE 1,1}

\subsection{Motivations of research}

Figure 2 shows an overview of some part of geological condition from a project in Jilin province of China. It should be noted that this sketch is only a rough estimation for involved tunnel, and each part of them may include subclass of rock types. The motivations of this paper can be boiled down to following aspects. In current site operation, the driver of TBM assesses the geological features by experience, and further chooses thrust force and torque to adjust the advance speed and control tunneling efficiency. But such a manner is a considerably conservative operation strategy, which will cause the advance rate remains at a low level. Thrust speed of TBM is related with the geological condition, such as surrounding rock strength, hardness, development degree, etc. Furthermore, the geology condition may be unknown and varying, as shown in Figure 2. This brings the difficulties in decision-making on control parameters setting. Finally, during the excavating, drivers should not only consider the excavate efficiency but also the economical performances degradation caused by abrasion of hobs in cutter-head. Besides, we should also care about risks on the safety and stationarity from cutter vibration. Therefore, it is vital to sufficiently consider the safety, efficiency, vibrancy and degeneracy during excavating and make a balance among these performances. This will benefit the adjustment of tunneling parameters to obtain a more efficient tunneling and enhance the automation level of TBM operation. Above issues impel us to study the optimization of control parameters under complex and varying geological conditions.

\section{Geological condition identification model}

This section will exploit the KNN algorithm to identify geological conditions based on the in situ sample data of several TBM crucial parameters. And considering the engineering requirements and the characteristics of the data samples, we modify the similarity measurement and voting methods in the KNN algorithm, such that making the algorithm more suitable for engineering and obtaining higher identification accuracy.

\subsection{Basic KNN algorithm}

$\mathrm{KNN}$ is a simple but effective algorithm that is widely used in data mining, especially for classification (Han et al., 2011). K-nearest neighbor means that the category of each sample can be represented by the $K$ samples closest to it. The key idea of the KNN algorithm is that if most of the $K$ nearest samples in the feature space of a sample belong to a certain category, then this sample should also belong to that category and have the same characteristics with the them. KNN algorithm is easy to implement with low time cost and desirable classification accuracy, which motivates us to exploit it in geological condition identification.

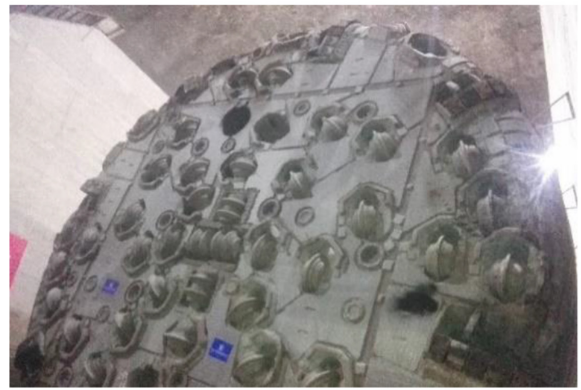

(a)

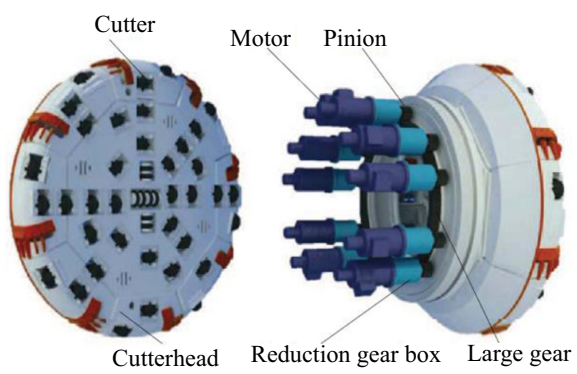

(b)
Plate 1.

The driving system: (a) real cutter-head in main structure 


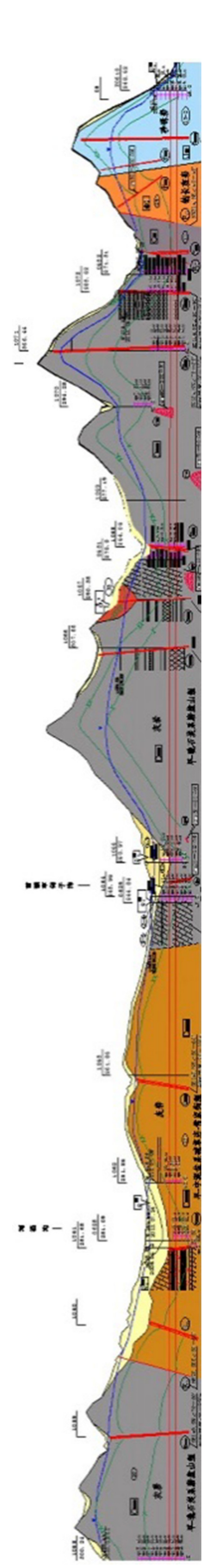

Optimization of TBM with geology recognition

91

Figure 2. The sketch of some part of geological condition in project 
JIMSE 1,1

\section{2}

The main steps are generally described as:

(1) Calculate the distance between the test data and corresponding training data;

(2) Implement sorting operation by increasing distance;

(3) Select $K$ points with the smallest distance;

(4) Determine the frequency of the first $K$ points in the category;

(5) Return the most frequent category as the predicted classification of test data.

It is worth noting that in the KNN algorithm, the dissimilarity among various objects is measured by calculating the distance among different samples. Commonly used distance measurements include:

(1) Manhattan distance

$$
d(x, y)=\sqrt{\sum_{k=1}^{n}\left|x_{k}-y_{k}\right|}
$$

(2) Euclidean distance

$$
d(x, y)=\sqrt{\sum_{k=1}^{n}\left(x_{k}-y_{k}\right)^{2}}
$$

(3) Minkowski distance

$$
d(x, y)=\sqrt[p]{\sum_{k=1}^{n}\left(x_{k}-y_{k}\right)^{p}}
$$

Specifically, when $p=\infty$, the measurement become the maximum of distance:

$$
L_{\infty}\left(x_{i}, y_{i}\right)=\max \left|x_{i}^{(l)}-y_{i}^{(l)}\right|
$$

After calculating the $K$ samples that are closest to the test samples, there are mainly two methods used to determine the category sample of the test samples, that is, the voting method and the weighted voting method. The voting method requires to select the category which is the largest number of samples from the $K$ samples that are closest to the test sample, and assigns its category to the test sample. The weighted voting method is to give different weights to the sample according to the distance of the $K$ samples from the test sample. The closer the distance to the test sample, the greater the weight. Finally, the voting results are weighted, and the category with the largest weight is assigned to the test sample as the final category. As for the selecting of $K$, one can refer to Han et al. (2011), Hall et al. (2008), Hassanat et al. (2014) and Zhang et al. (2017).

\subsection{Geological condition identification based KNN}

As a large-scale integrated system, TBM will generate amounts of data from many installed sensors. In this paper, we choose several crucial TBM parameters as features used in KNN algorithm, according to the expertise and observation from in situ data. The selecting TBM parameters includes rotation speed of cutter-head $\omega$, currents in driving motors $I$, total thrust force $F$, torque of cutter-head $T$, penetration rate $P$ and advance speed $v$. 
137,645 samples are selected for executing identification algorithm, where 100,000 samples for training and 37,645 samples for testing. The comparison results among KNN, C4.5 decision tree (Hssina et al., 2014) and PSO-SVM (Huang and Dun, 2008) are shown in Table 1. It is noted that KNN algorithm belongs to lazy algorithm, which has no explicit training process. Thus, we do not count the time cost in training of KNN in Table 1. From the results shown in Table 1 , the identification accuracy of decision tree is slightly higher than $\mathrm{KNN}$ algorithm, but takes much time in training.

Since new geological conditions will be encountered during the excavation process, this requires the algorithm to be able to adapt to the changes in new surroundings. Thus, the model needs to be updated many times during the construction process. As can be seen from Table 1, although the decision tree algorithm is a bit higher in accuracy than the KNN algorithm, it spends more time in the training process. In order to achieve online identification, the KNN algorithm is selected as the basic algorithm of the geological condition identification. In what follows, we focus on how to improve the accuracy of identification based on the KNN method according the situation of engineering.

The modifications mainly include the adjustment of similarity measurement and the weights regulation in voting procedure of $\mathrm{KNN}$ algorithm. In above verification, we used the common Euclidean distance as the similarity measurement. It is easy to find that the importance degree of each feature in this measurement is the same (that is, the weight coefficients are the same). However, in practical, the impact on each parameter is varied with geological condition. Therefore, in order to improve accuracy, we should give higher priority to those features in similarity measurement, which are easier to be affected by geological condition. To this end, we increase the weights of the cutter-head, torque and total thrust in the distance measurement, respectively. The new similarity measurement is:

$$
d=\sqrt{1.2\left(T_{x}-T_{y}\right)^{2}+1.1\left(F_{x}-F_{y}\right)^{2}+\left(I_{x}-I_{y}\right)^{2}+\left(v_{x}-v_{y}\right)^{2}+\left(\omega_{x}-\omega_{y}\right)^{2}+0.8\left(P_{x}-P_{y}\right)^{2}}
$$

Furthermore, the voting procedure in $\mathrm{KNN}$ algorithm has used equal-voting manner before. However, owing to the amount of each rock types is different in samples, it is likely to cause that most of the votes tend to be the type which has large numbers of sample among them. Therefore, in voting procedure, it is necessary to reduce the weights of certain sample types, so that voting become more reasonable and suitable for practical engineering. According to the observation from sample, the revised geological condition weights in voting procedure of $\mathrm{KNN}$ algorithm are shown in Table 2 . The cross notation means that there are not these types

\begin{tabular}{lccc}
\hline Algorithm & KNN & C4.5 decision tree & PSO-SVM \\
\hline Accuracy & $83.7 \%$ & $84.9 \%$ & $64.6 \%$ \\
Time cost in training & - & $130 \mathrm{~s}$ & $60 \mathrm{~s}$ \\
\hline
\end{tabular}

Table 1.

Comparison results with other algorithms

\begin{tabular}{llllll}
\hline Rock types & II & III & IV & V & \\
\hline Granite & 1.1 & 1 & $\times$ & $\times$ & \\
Tuff & 1 & 1 & 1 & $\times$ & Table 2. \\
Diorite & 1 & 1 & $\times$ & $\times$ & Weight adjustment \\
Limestone & 1 & 0.85 & 0.9 & 1 & table for voting \\
Glutenite & $\times$ & 1 & 1 & 1.1 & procedure \\
Mudstone & $\times$ & $\times$ & $\times$ & & \\
\hline
\end{tabular}


JIMSE 1,1

\section{4}

of rock in data set mentioned above, while the rest with values means that these rock types appear in used data sample.

In order to verify the efficacy of identification with above modification, we retest above samples covering various rock types. Table 3 only shows some parts of identification results based on KNN algorithm after modification in weights setting. It should be noted that the value like " $2 / 3$ " in Table 3 , represents the second type II of the third class surrounding rock. If the label in column "Prediction" is the same with the one in column "Real", that means identification is correct. The accuracy of identification reaches $96.1 \%$ over above all data samples based on KNN algorithm with proposed modification. Compared with the identification result before $(83.7 \%)$, the identification accuracy using modified algorithm has dramatically improved.

\section{The multi-objective optimization problem formulation}

Tunneling in complex geology, TBM needs to consume a huge amount of energy, mainly caused by the cutter-head system and the hydraulic system. These energies provide power for machine to conduct rotation and advancement. Besides the energy consumption, we should also consider the cutter hob wear and the cutter vibration during the excavating. The target of control is to make the excavating process fast at a high tunneling rate. On the other hand, the driver of TBM manually adjusts the advance speed and cutter-head rotate speed to adapt to the geological conditions in practical. If TBM is working under homogeneous rock, the advance speed and cutter-head rotate speed is tuned to be bigger so that accelerating the excavation process. However, the cutter hob abrasion will increase along with the increasing of the rotate speed, due to the limited load that the cutter hob be able to bear. At the same time, the vibration stemmed from cutter-head can be transmitted to other parts of the machine, which may damage other equipment or physical devices. Therefore, the reasonable settings of advance speed and cutter head rotate speed is of crucial importance to the whole machine. It should be noted here that different performances of TBM have different priorities in practical construction. At present, this kind of priority is summarized as experience

\begin{tabular}{|c|c|c|c|c|c|c|c|c|}
\hline $\begin{array}{l}\text { Sample } \\
\text { number }\end{array}$ & Prediction & Real & $\begin{array}{l}\text { Sample } \\
\text { number }\end{array}$ & Prediction & Real & $\begin{array}{l}\text { Sample } \\
\text { number }\end{array}$ & Prediction & Real \\
\hline 253 & $2 / 2$ & $2 / 2$ & 854 & $4 / 5$ & $4 / 5$ & 4,761 & $1 / 3$ & $1 / 3$ \\
\hline 254 & $2 / 2$ & $2 / 2$ & 855 & $4 / 5$ & $4 / 5$ & 4,762 & $1 / 3$ & $1 / 3$ \\
\hline 255 & $2 / 2$ & $2 / 2$ & 856 & $4 / 5$ & $4 / 5$ & 5,633 & $4 / 4$ & $4 / 4$ \\
\hline 256 & $2 / 2$ & $2 / 2$ & 857 & $4 / 5$ & $4 / 5$ & 5,634 & $4 / 4$ & $4 / 4$ \\
\hline 257 & $2 / 2$ & $2 / 2$ & 1,451 & $4 / 2$ & $4 / 2$ & 5,635 & $4 / 4$ & $4 / 4$ \\
\hline 258 & $2 / 3$ & $2 / 2$ & 1,452 & $4 / 2$ & $4 / 2$ & 5,636 & $4 / 4$ & $4 / 4$ \\
\hline 259 & $2 / 3$ & $2 / 3$ & 1,453 & $4 / 2$ & $4 / 2$ & 5,637 & $4 / 4$ & $4 / 4$ \\
\hline 260 & $2 / 3$ & $2 / 3$ & 1,554 & $4 / 2$ & $4 / 2$ & 5,638 & $4 / 4$ & $4 / 4$ \\
\hline 261 & $2 / 3$ & $2 / 3$ & 3,067 & $3 / 3$ & $3 / 3$ & 5,639 & $4 / 5$ & $4 / 4$ \\
\hline 262 & $2 / 3$ & $2 / 3$ & 3,068 & $3 / 3$ & $3 / 3$ & 7,991 & $4 / 2$ & $4 / 2$ \\
\hline 263 & $2 / 3$ & $2 / 3$ & 3,069 & $3 / 2$ & $3 / 3$ & 7,992 & $4 / 2$ & $4 / 2$ \\
\hline 264 & $4 / 3$ & $4 / 3$ & 3,070 & $3 / 3$ & $3 / 3$ & 7,993 & $4 / 2$ & $4 / 2$ \\
\hline 265 & $4 / 3$ & $4 / 3$ & 3,071 & $3 / 3$ & $3 / 3$ & 7,994 & $4 / 2$ & $4 / 2$ \\
\hline 266 & $4 / 3$ & $4 / 3$ & 3,072 & $3 / 3$ & $3 / 3$ & 7,995 & $4 / 2$ & $4 / 2$ \\
\hline 267 & $4 / 3$ & $4 / 3$ & 3,073 & $3 / 3$ & $3 / 3$ & 7,996 & $4 / 2$ & $4 / 2$ \\
\hline 268 & $4 / 3$ & $4 / 3$ & 3,074 & $3 / 3$ & $3 / 3$ & 7,997 & $4 / 2$ & $4 / 2$ \\
\hline 269 & $4 / 3$ & $4 / 3$ & 4,758 & $1 / 3$ & $1 / 3$ & 7,998 & $4 / 2$ & $4 / 2$ \\
\hline 270 & $4 / 3$ & $4 / 3$ & 4,759 & $1 / 3$ & $1 / 3$ & 7,999 & $4 / 2$ & $4 / 2$ \\
\hline 271 & $4 / 3$ & $4 / 3$ & 4,760 & $1 / 3$ & $1 / 3$ & 8,000 & $4 / 2$ & $4 / 2$ \\
\hline
\end{tabular}
prac
Table 3. 267

Some parts of results 268 on geological condition 269 identification after $\quad 270$ modification 
knowledge by experts or drivers. For instance, the energy performance has the lowest priority, because in practice it is likely to give priority to other factors, with the permission to have a relatively large energy consumption.

In this section, a multi-objective model is established, under the considerations of tunneling rate, cutter hob wear, cutter-head vibration and energy consumption. Moreover, the proposed multi-objective optimization problem will be transformed into a single-objective optimization problem for the convenience of solving. Then the optimization problem will be solved by using genetic algorithm, to obtain the optimal advance speed and cutter-head rotate speed, which trades off among abovementioned performances.

\subsection{Penetration rate}

Penetration rate is often referred to measure the ability of TBM to excavate on the hard rock. In practical construction, intuitively and ideally, increasing the rotate speed of cutter-head and advance speed can make TBM to excavate more quickly. Let $v$ and $\omega$ be advance speed and rotate speed of cutter-head, respectively. The construction efficiency can be described by the penetration rate which defined as:

$$
\eta=\frac{v}{\omega}
$$

This index reflects the amount of excavating within each rotation of cutter-head. Note that, there are constraints on $v$ and $\omega$ because of the limitation from physical elements. Furthermore, increasing them is not necessarily to get a desirable or better performance in practical engineering.

\subsection{Hob cutters wear}

The abrasion of hob (see Figure 3 ) is a serious issue for TBM during excavating especially working in hard rock condition. Changing hob frequently will not only slow down the schedule of the whole construction but also increase the cost (Hassanpour, 2018). Therefore, it is necessary to consider how to reduce the abrasion of hobs so that prolong the working life of them.

The calculation of hob wears is mainly based on the analysis of rock broken mechanism, material properties of cutting tool and the forces on hobs. As for the computation of the forces on hob, the model CSM (Colorado School of Mines, CSM) is widely adopted in many theoretical research studies and practical engineering (Rostami et al., 1996, 2002). According to the penetration, rock parameters and cutting tool parameters, one can obtain rolling force, vertical force and resultant force. The CSM model is described as

$$
\begin{aligned}
F_{t d} & =\int_{0}^{\phi} P T R d \omega=\int_{0}^{\phi} \operatorname{PTR}\left(1-\frac{\varphi}{\phi}\right)^{\psi} d \varphi=P T R \int_{0}^{\varphi}\left(1-\frac{\varphi}{\phi}\right)^{\psi} d \varphi \\
& =C^{3} \sqrt{\frac{8}{\phi \sqrt{R T}} \sigma_{c}^{2} \sigma_{s}} \frac{T R \phi}{1+\psi}
\end{aligned}
$$

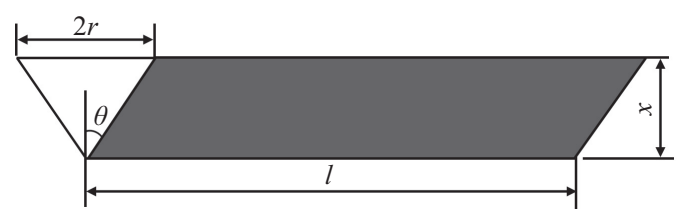

Figure 3. Schematic diagram of hob
Optimization of TBM with geology recognition 
JIMSE 1,1

$$
\begin{gathered}
\phi=\operatorname{arccoss}\left(\frac{R-p}{R}\right) \\
\eta=\frac{v}{\omega}
\end{gathered}
$$

where $F_{t d}$ is the resultant force on cutter hob, $R$ represents the radius of cutter-head, $\psi$ is pressure distribution coefficient. $T$ and $\phi$ represent the hob blade width and angle, respectively. $P$ denotes the broken pressure that can be calculated according to rock compressive strength, shear strength, the size of the hob and some other parameters. $\eta$ denotes the penetration rate.

Decomposing the force on cutter hob, the rolling force $F_{r}$ and the vertical force $F_{t}$ can be obtained as follows:

$$
\begin{aligned}
& F_{r}=P T R \int_{0}^{\theta}\left(1-\frac{\omega}{\phi}\right)^{\psi} \sin \varphi d \varphi=F_{t d} \sin \frac{\phi}{2} \\
& F_{t}=P T R \int_{0}^{\theta}\left(1-\frac{\omega}{\phi}\right)^{\psi} \cos \varphi d \varphi=F_{t d} \cos \frac{\phi}{2}
\end{aligned}
$$

Usually the penetration depth is much smaller than the radius of cutter, so the term $\sin (\phi / 2)$ is too small to be omitted. Thus, the force on cutter hob is mainly composed by the vertical force.

The radial wears of hob after rotating a circle can be computed by (12)-(14):

$$
\begin{gathered}
X_{0}=\frac{Q_{0} l_{0}}{\pi D_{0} T} \\
l_{0}=R \phi \approx \sqrt{D_{0} \eta} \\
Q_{0}=K_{s} \frac{F_{t}}{\pi \sigma_{s}}
\end{gathered}
$$

where $l_{0}$ is the sliding distance of one circle, $D_{0}$ is hob diameter, $Q_{0}$ is the abrasion per sliding distance, $K_{s}$ is wear coefficient, $\sigma_{s}$ is yield strength of cutting tool. The wear rate is defined by:

$$
k=\frac{X}{L}=\frac{X_{0} R_{i} / R}{p}
$$

where $R_{i}, i=1, \ldots, N$, represents install radius of the $i$-th cutter hob, $X$ denotes the abrasion loss within the driving distance $L$ of TBM. Let $N$ be the number of hob, and suppose that the force and torque on cutter-head are uniformly distributed. Then we can obtain the force and torque on cutter-head computed by equation (16) and (17), respectively.

$$
\begin{aligned}
& F_{n}=N F_{t d} \cos (\phi / 2) \\
& T=N \frac{D}{2} F_{t d} \sin (\phi / 2)
\end{aligned}
$$

Through further transforming, the compressive strength of rock can be formulated as:

$$
\lg \sigma_{c}=\frac{1}{2}\left\{\lg \left(F_{t d}\right)-\lg \left(\frac{C^{3} \sqrt{\frac{s}{\Phi \sqrt{R T}} \Phi R T}}{1+\psi}\right)-\lg \sigma_{s}-\lg N\right\}
$$


Therefore, based on the above analysis, the wear rate of the hob is related with material of cutting tool, cutting tool radius, distance among the hobs, compressive strength of rock, penetration, thrust force and cutter-head torque, etc.

\subsection{Cutter-head vibration}

The energy caused by cutter-head vibration can be transmitted through multiple path to other components, and may cause a large amount energy gathered in some local components which may be enough to damage them. In this paper, we consider vibration intensity as the root mean square value of the average vibration velocity of each measuring point on the cutter-head from three directions. Then, the vibration intensity can be measured by the maximum or average vibration velocity from different directions.

There are three sensors located in different positions of cutter-head. Once the average and maximum vibration velocity of these positions are measured, the vibration severity can be calculated according to equation (19) and (20).

$$
\begin{gathered}
v_{r m s}=\sqrt{\frac{1}{T} \int_{0}^{T}|\vec{v}|^{2}}(t) d t=\sqrt{\frac{1}{T} \int_{0}^{T}\left[v_{x}^{2}(t)+v_{y}^{2}(t)+v_{z}^{2}(t)\right] d t} \\
v_{\max }=\sqrt{\frac{1}{T} \int_{0}^{T}|\vec{v}|^{2}}(t) d t=\sqrt{\frac{1}{T} \int_{0}^{T}\left[v_{x, \text { max }}^{2}(t)+v_{y, \text { max }}^{2}(t)+v_{z, \text { max }}^{2}(t)\right] d t}
\end{gathered}
$$

In practical, (21) and (22) are exploited with $N$ discrete points due to the vibration velocity signals are sampled at a certain time interval.

$$
\begin{gathered}
v_{r m s}=\sqrt{\frac{1}{N} \sum_{n=0}^{N-1}\left[v_{x}^{2}(n)+v_{y}^{2}(n)+v_{z}^{2}(n)\right]} \\
v_{\max }=\sqrt{\frac{1}{N} \sum_{n=0}^{N-1}\left[v_{x, \max }^{2}(n)+v_{y, \max }^{2}(n)+v_{z, \max }^{2}(n)\right]}
\end{gathered}
$$

According to the vibration degree given by ISO organization, the relationships between tunnel parameters and vibration degree can be estimated, as shown from Figures 4-6.

Finally, based on above analysis and discrete sample data, it is easy to estimate the relationships between vibration intensity and tunneling parameters considered in this paper. It can be seen from Figure 6 that the trend can be approximated by an inverted parabolic, whose parameters are estimated through curve fitting implemented in Matlab. Then the estimated relationships between vibration and penetration can be shown as follows.

$$
\begin{aligned}
& V i b_{\max }=-0.44 p^{2}+8.77 p+7.2 \\
& V i b_{r m s}=-0.13 p^{2}+2.88 p-1.3
\end{aligned}
$$

\subsection{Driving energy consumption}

The TBM tunneling process will consume a large amount of energy, including the energy used for breaking rock, supporting hydraulic cylinder, propelling the machine and slagging 


\section{JIMSE 1,1}

98

Figure 4.

Relationship of rotate speed and vibration severity

\section{Figure 5.}

Relationship of advance speed and vibration severity

Figure 6.

Relationship between penetration and vibration severity
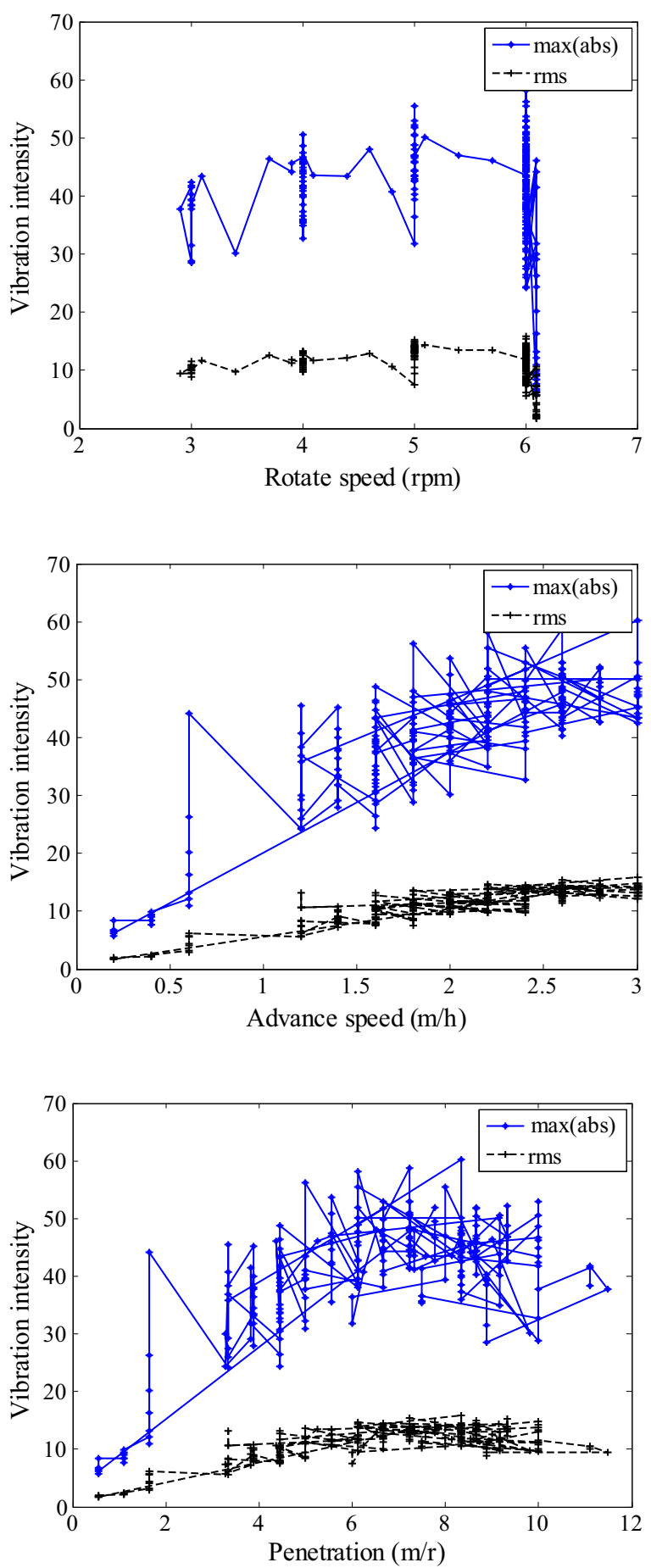
out the rubbles. Among them, the energy costs in cutter-head system and hydraulic thrust system are the largest part of them, which can be calculated by

$$
\begin{gathered}
E=E_{1}+E_{2} \\
E_{1}=\omega \sum_{m=1}^{N} T_{m}=\omega T(v, \omega) \\
E_{2}=v \sum_{m=1}^{2} F_{x}=v F_{n}(v, \omega)
\end{gathered}
$$

where $E_{1}$ represents the energy consumed by cutter-head system to break the rock, $E_{2}$ is the energy consumed by hydraulic thrust system to push the TBM machine forward, $\omega$ represents cutter-head rotate speed and $v$ is the advance speed, $T$ and $F$ denote the cutter-head torque and the thrust force, respectively, which can be calculated by (16) and (17).

The analysis of this section shows that, the energy consumed by TBM has closely connection to the several crucial tunneling parameters. Different choose of these tunnel parameters will make different influences on the energy consumption. Therefore, the evaluation function (25)-(27) can be used to reflect the energy consumption level of TBM.

\subsection{Tunneling risk}

The works in (Hong et al., 2009) provided a quantitative risk evaluation based on event tree analysis technique during TBM operating. However, the proposed strategy is not for the TBM control but for the preparatory work before excavating. Sousa and Einstein (2012) have built a risk analysis during tunnel construction using Bayesian network. The results are not used directly in excavating but providing supports to experts for decision-making. Different from above works, this paper proposes a tunneling risk performance, which will be used in optimization of control parameters for TBM during excavating. Specifically, by counting the number of abnormal working conditions under various geological conditions, we endow a risk factor to each geological condition. More specifically, we estimate the probability that an abnormal situation may occur in the TBM equipment under corresponding geological condition, and the tunneling risk can be calculated by

$$
P=\max \left(\frac{x_{\max }-x_{i}}{x_{\max }-x_{\min }}\right) p G
$$

where $x_{i}$ is the working parameters of TBM, $p_{G}$ is the probability that abnormal situation occurs under a certain geological condition.

\subsection{Multi-objective optimization problem}

There are many methods to solve multi-objective optimization problems, wherein Pareto method and single-objective method have been two widely adopted currently. For the former, one can refer to Giagkiozis and Fleming (2015) and the literatures therein. In this paper, the multi-objective problem will be converted into a single-objective problem in a way that integrates multiple optimization functions as a single function in a certain manner, such that the original multi-objective optimization problem is equivalent or approximated to the new one, see Konak et al. (2006) and Marler and Arora (2004). The most popular manner is to make single-objective function as a linear combination of multiple objective function. More specifically, the optimization problem can be formulated as
Optimization of TBM with geology recognition 


\section{JIMSE 1,1}

\section{0}

$$
\begin{gathered}
\min F(x)=\sum_{i=1}^{s} \alpha_{i} f_{i}(x) \\
\text { s.t. } a)\left[F_{n} T\right]=h(v, \omega) \\
\text { b) } V_{\min }<v<V_{\max } \\
\text { c) } F_{\min }<F<F_{\max } \\
\text { d) } \omega_{\min }<\omega<\omega_{\max } \\
\text { e) } T_{\min }<T<\max
\end{gathered}
$$

where $f_{i}(x)$ is $i$-th performance function of TBM, $\alpha_{i}$ is the weight of corresponding performance, $s$ is the number of performance functions to be considered. Constraint (a) denotes the dynamics constraint, which can be found in Li et al. (2013). Constraint (b)-(e) are hard constraints of TBM that cannot be violated, including the bounds of advancing speed, thrust force, rotation speed of cutter-head and total toque. The selection of weighted factors for different objective functions will have a decisive significance for the final optimization result, due to the fact that the weight setting will affect the search direction of the optimization algorithm. Therefore, the problem boils down to how to determine the optimal weighted factors.

Different performance functions may have different requirements under different geological conditions (Bäppler, 2016). Therefore, it is necessary to assign different weights for each performance function in the multi-objective optimization model under corresponding geological condition. By setting suitable weights for each objective performance functions, decision-making will be implemented with geological adaptability.

The weight setting of objective function under different geological conditions is established thought summarizing relevant construction experiences. According to the overall situation of rock indexes and surrounding rocks, the geological conditions can be divided into the following four categories:

(1) Relatively complete homogeneous hard rock;

(2) Medium hard rock with relatively developed joints;

(3) Uneven rocks with soft and hard rock with developed joints;

(4) Fault fracture zone and bad geology.

These four types of geological conditions have distinct characteristics in the construction process caused by properties and features of surrounding rock. The match relationships between rock types and corresponding geological conditions is illustrated in Table 4, followed by the descriptions of setting rules for weights.

(1) Relatively complete homogenous hard rock

Table 4.

Weight distribution under four geological conditions
Rock type

Granite II, Tuff II

Granite III, Tuff III, Diorite II, Limestone II, Limestone III Tuff IV, Diorite III, Limestone IV, Glutenite III, Glutenite IV Glutenite V, Mudstone V

Note(s): (1) Relatively complete homogenous hard rock; (2) Hard rock with developed joints; (3) Uneven hard and soft rocks with developed joints; (4) Fault fracture zone 
Under this geological condition, the geological condition is so well that TBM can excavate rapidly. In this case, we can give the excavation efficiency a higher weight. In addition, the wear of the cutter-head hobs during the rotation should be concerned with a high priority due to the equipment is operating at a high-speed level. Thus, the weight of cutter-head wear should be increased correspondingly. Besides, the probability that occurs abnormal working situation or accident is relatively small, so the weight on risk of the tunneling can be set as the lowest level.

(2) Hard rock with more developed joints

Under this geological condition, rapid excavation is generally possible. As the joint is developed, it will cause uneven force on the cutter-head. When TBM encounter with this kind of situation, the cutter-head is prone to generate strong vibration caused by uneven stress. At this moment, it is necessary to give high weight to the efficiency and vibration performance while increasing the risk level.

(3) Uneven hard and soft rocks are developed in joints

In this case, joints are fully developed. Strong shaking will occur during the excavation process, so the vibration needs to be considered as the first factor. Furthermore, the progress of the excavation is not recommended to be fast, cause the stability of the surrounding rock and risk increase under this geological condition.

(4) Fault fracture zone and bad geological sections

Under such geological conditions, one do hope to avoid potential collapse and unpredictable accidents by controlling the operating parameters gently, rather than pursuing the tunneling efficiency of the equipment.

After above analysis, a group of weights setting for optimization functions is summarized and listed in Table 5, which will be used in optimization problem-solving later.

\section{Simulation}

\subsection{Genetic algorithm for multi-objective optimization problem}

This paper adopts genetic algorithm to solve the optimization problem. Genetic algorithm is a method that uses random search to perform optimization, which does not require the continuity and derivability of the objective function. It uses a probabilistic optimization method to automatically optimize the search space and search direction. Genetic algorithm and its variants have been widely applied in the fields of combinatorial optimization, machine learning, adaptive control, etc., because of global optimization ability. This paper will not describe the genetic algorithm in detail due to the limitation of space. More details and extensions can be found in Guerrero et al. (2018) and Kramer (2017). Here we only briefly illustrate general procedures of genetic algorithm.

\begin{tabular}{lccccc}
\hline Geological conditions & Efficiency & Energy consumption & Abrasion & Vibration & Construction risk \\
\hline$(1)$ & 0.4 & 0.01 & 0.3 & 0.1 & 0.1 \\
$(2)$ & 0.3 & 0.01 & 0.1 & 0.3 & 0.15 \\
$(3)$ & 0.2 & 0.03 & 0.1 & 0.3 & 0.2 \\
$(4)$ & 0.1 & 0 & 0.1 & 0.1 & 0.3
\end{tabular}

Note(s): (1) Relatively complete homogenous hard rock; (2) Hard rock with developed joints; (3) Uneven hard and soft rocks with developed joints; (4) Fault fracture zone
Optimization

of TBM with geology recognition

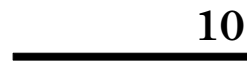


JIMSE 1,1

\section{2}

Figure 7.

The optimization results under Granite II
The outline of procedures in basic genetic algorithm can be summarized as follows:

(1) Initialization: Generate random population of $n$ chromosomes;

(2) Individual assessment: Evaluate the fitness $f(x)$ of each chromosome $x$ in the population;

(3) New population: Create a new population by repeating following steps until the new population is complete:

- Selecting operation: Select two parent chromosomes from a population according to their fitness (the better fitness, the bigger chance to be selected);

- Crossover operation: With a crossover probability cross over the parents to form new offspring (children). If no crossover was performed, offspring is the exact copy of parents;

- Mutation operation: With a mutation probability mutate new offspring at each locus (position in chromosome);

- Accepting: Place new offspring in the new population;

(4) Test: If the end condition is satisfied, stop and return the best solution in current population; otherwise, go to step 2.

\subsection{Simulation results}

All related parameters mentioned in this paper are draw from the in situ data of project. To verify our proposed method, we select the data on certain day in December 2015. Figure 7
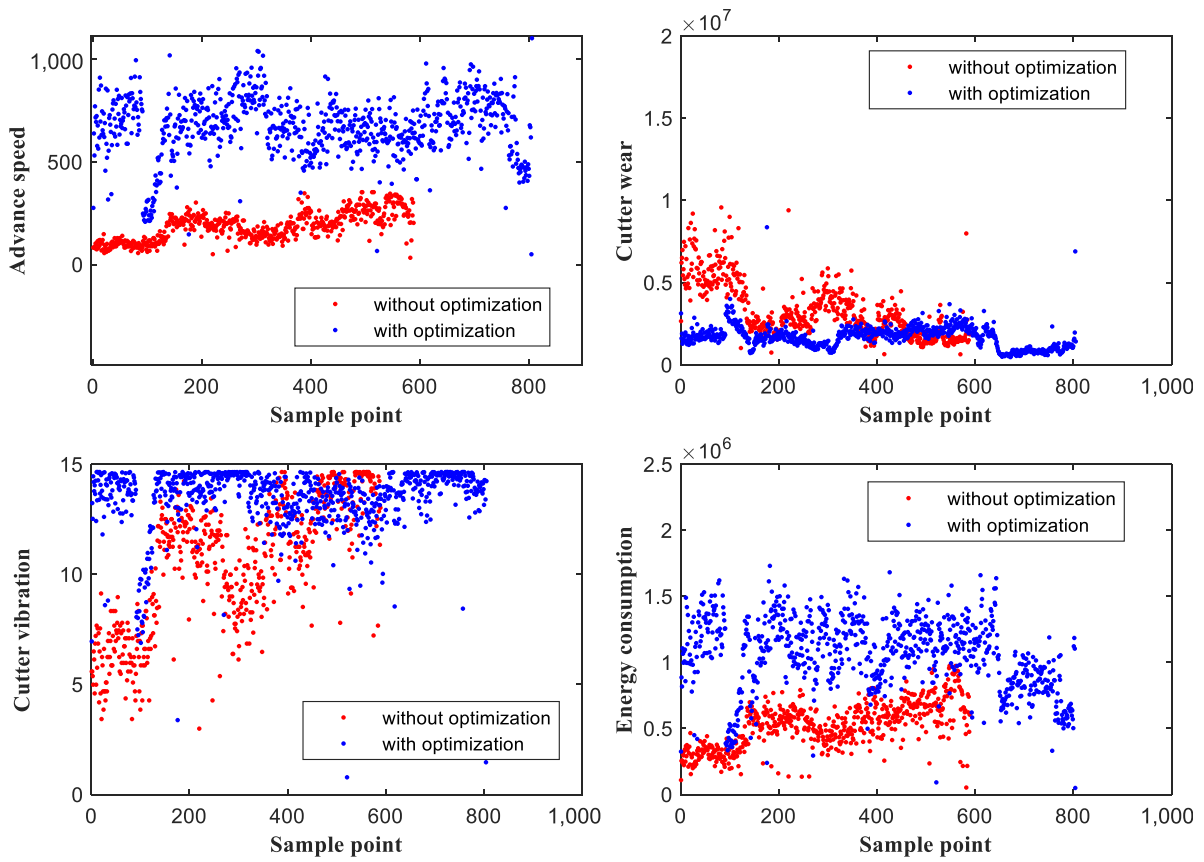

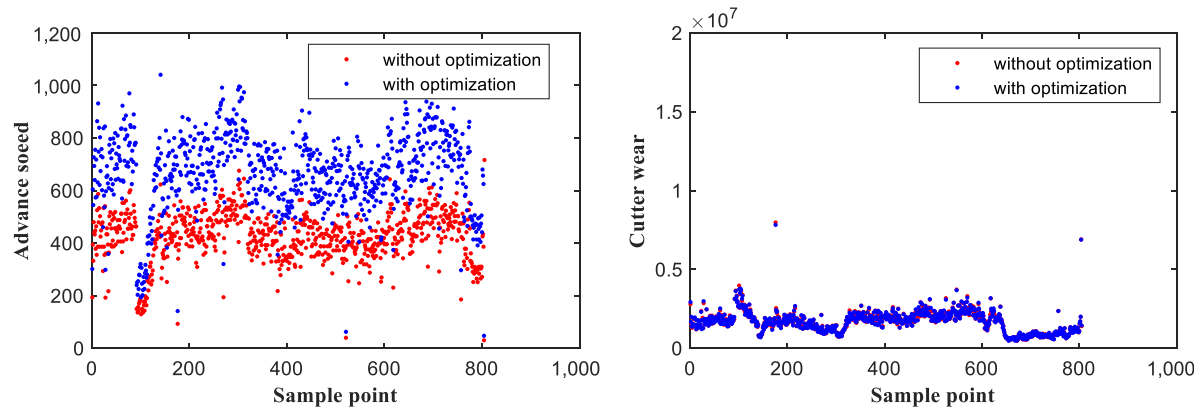

Optimization

of TBM with geology

recognition
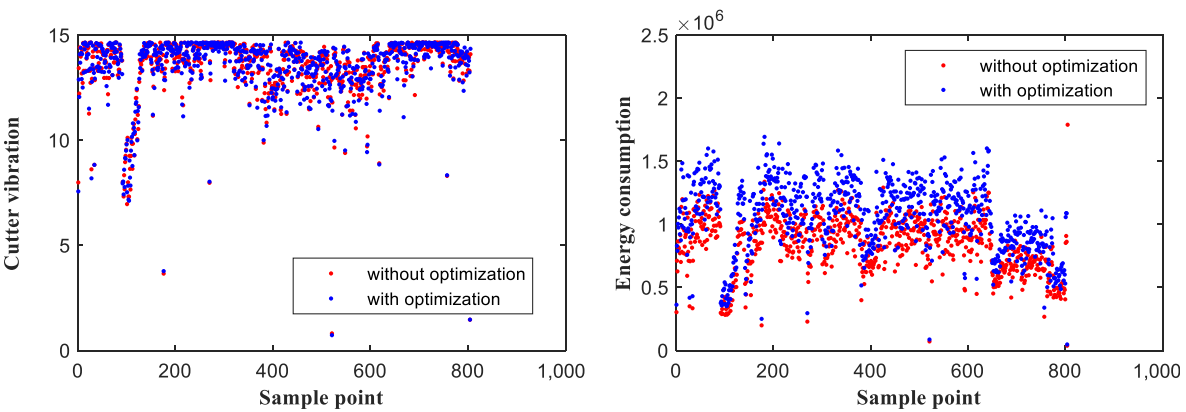

103

shows the optimization results under granite II. Under this kind of geology, the advance speed was increased after optimization while the cutter wear was reduced. In should be noted that the energy consumption is higher than before optimization shown in Figure 7. As mentioned in Section 4, the importance degree of energy consumption is lowest compared with other TBM performances. This consideration is based on the fact that, in actual construction, the progress of TBM tunneling and the security have a higher priority to be concerned. That is, we want to speed up the advance progress while not rising the vibration and hob wear so much, and it is allowed that even the energy consumption increases to a certain extent. Thus, the increment in cutter vibration and energy consumption here is acceptable for practice.

Figure 8 shows the optimization results under diorite III, which has the similar effectiveness with Figure 7. Under diorite III, the advance speed was enhanced after optimization while keeping the vibration and wear almost invariant at the price of increasing energy consumptions slightly. The same phenomenon can also be found in other type of geology, which verifies the efficacy and suitability of proposed method.

\section{Conclusion}

This paper designed a multi-objective optimization control scheme for TBM. In the early stage, an improved KNN algorithm based on actual engineering data, were used to identify the types of geological rocks with a high prediction accuracy. Secondly, several vital performance functions were provided and utilized as the objective functions in the optimization problem. The multi-objective optimization problem for obtaining the optimal control parameters in the TBM tunneling process was further established. The problem was transformed into a single-objective optimization problem and solved by genetic algorithm. The results show that the proposed method can effectively increase the efficiency of tunneling. 


\section{JIMSE 1,1}

\section{References}

Bäppler, K. (2016), "New developments in TBM tunnelling for changing grounds", Tunnelling and Underground Space Technology, Vol. 57, pp. 18-26.

Gao, X., Shi, M., Song, X., Zhang, C. and Zhang, H. (2019), "Recurrent neural networks for real-time prediction of TBM operating parameters", Automation in Construction, Vol. 98, pp. 225-235.

Giagkiozis, I. and Fleming, P.J. (2015), "Methods for multi-objective optimization: an analysis", Information Sciences, Vol. 293, pp. 338-350.

Gong, Q., Yin, L., Ma, H. and Zhao, J. (2016), "TBM tunnelling under adverse geological conditions: an overview", Tunnelling and Underground Space Technology, Vol. 57, pp. 4-17.

Guerrero, C., Lera, I. and Juiz, C. (2018), "Genetic algorithm for multi-objective optimization of container allocation in cloud architecture", Journal of Grid Computing, Vol. 16 No. 1, pp. 113-135.

Hall, P., Park, B.U. and Samworth, R.J. (2008), "Choice of neighbor order in nearest-neighbor classification", The Annals of Statistics, Vol. 36 No. 5, pp. 2135-2152.

Han, J., Pei, J. and Kamber, M. (2011), Data Mining: Concepts and Techniques, Elsevier.

Hassanat, A.B., Abbadi, M.A., Altarawneh, G.A. and Alhasanat, A.A. (2014), "Solving the problem of the K parameter in the KNN classifier using an ensemble learning approach", arXiv preprint arXiv, 1409.0919.

Hassanpour, J. (2018), "Development of an empirical model to estimate disc cutter wear for sedimentary and low to medium grade metamorphic rocks", Tunnelling and Underground Space Technology, Vol. 75, pp. 90-99.

Home, L. (2016), "Hard rock TBM tunneling in challenging ground: developments and lessons learned from the field", Tunnelling and Underground Space Technology, Vol. 57, pp. 27-32.

Hong, E.S., Lee, I.M., Shin, H.S., Nam, S.W. and Kong, J.S. (2009), “Quantitative risk evaluation based on event tree analysis technique: application to the design of shield TBM", Tunnelling and Underground Space Technology, Vol. 24 No. 3, pp. 269-277.

Hssina, B., Merbouha, A., Ezzikouri, H. and Erritali, M. (2014), "A comparative study of decision tree ID3 and C4. 5", International Journal of Advanced Computer Science and Applications, Vol. 4 No. 2, pp. 13-19.

Huang, C.L. and Dun, J.F. (2008), "A distributed PSO-SVM hybrid system with feature selection and parameter optimization”, Applied Soft Computing, Vol. 8 No. 4, pp. 1381-1391.

Konak, A., Coit, D.W. and Smith, A.E. (2006), "Multi-objective optimization using genetic algorithms: a tutorial", Reliability Engineering and System Safety, Vol. 91 No. 9, pp. 992-1007.

Kramer, O. (2017), Genetic Algorithm Essentials, Vol. 679, Springer.

Li, X., Yu, H., Yuan, M. and Zhao, Y. (2013), "Research on dynamic models and performances of shield tunnel boring machine cutterhead driving system", Advances in Mechanical Engineering, Vol. 5, p. 359757.

Liu, R., Sun, J.Z., Luo, Y.Q., Sun, W. and Li, W.D. (2011), "Research on multi-motor synchronization control based on the ring coupling strategy for cutterhead driving system of shield machines", Applied Mechanics and Materials, Vols 52-54, pp. 65-72.

Marler, R.T. and Arora, J.S. (2004), "Survey of multi-objective optimization methods for engineering", Structural and Multidisciplinary Optimization, Vol. 26 No. 6, pp. 369-395.

Rostami, J., Ozdemir, L. and Nilson, B. (1996), "May. Comparison between CSM and NTH hard rock TBM performance prediction models", Proceedings of Annual Technical Meeting of the Institute of Shaft Drilling Technology, Las Vegas, pp. 1-10.

Rostami, J., Gertsch, R. and Gertsch, L. (2002), "Rock fragmentation by disc cutter: a critical review and an update", Proceedings of North American Rock Mechanics Symposium (NARMS'02), Toronto, Ontario. 
Rostami, J. (2016), "Performance prediction of hard rock Tunnel Boring Machines (TBMs) in difficult ground", Tunnelling and Underground Space Technology, Vol. 57, pp. 173-182.

Sousa, R.L. and Einstein, H.H. (2012), "Risk analysis during tunnel construction using Bayesian Networks: porto Metro case study", Tunnelling and Underground Space Technology, Vol. 27 No. 1 , pp. 86-100.

Optimization of TBM with geology recognition

Zhang, S., Li, X., Zong, M., Zhu, X. and Cheng, D. (2017), "Learning k for knn classification”, ACM Transactions on Intelligent Systems and Technology (TIST), Vol. 8 No. 3, pp. 1-19.

Zhao, Y., Wang, J., Zhang, L. and Hu, T. (2015), "August. Fuzzy-PID based induction motor control and its application to TBM cutter head systems", International Conference on Intelligent Robotics and Applications, Springer, Cham, pp. 511-522.

Zheng, Y.L., Zhang, Q.B. and Zhao, J. (2016), "Challenges and opportunities of using tunnel boring machines in mining”, Tunnelling and Underground Space Technology, Vol. 57, pp. 287-299.

\section{Corresponding author}

Hongyuan Wang can be contacted at: wanghongyuan@sjtu.edu.cn

For instructions on how to order reprints of this article, please visit our website:

www.emeraldgrouppublishing.com/licensing/reprints.htm

Or contact us for further details: permissions@emeraldinsight.com 